\title{
Study on laser-based white light sources
}

\author{
Ee-Ning Ooi ${ }^{\mathrm{a}}$, Xiaobin Sun ${ }^{\mathrm{a}}$, Boon S. Ooi ${ }^{\mathrm{a}}$, Tien Khee $\mathrm{Ng}^{\mathrm{a}}$, and Chao Shen ${ }^{* a, b}$, \\ aphotonics Laboratory, King Abdullah University of Science and Technology (KAUST), 4700 \\ KAUST, Thuwal, Saudi Arabia 21534; ' SaNoor Technologies, KAUST Innovation Cluster, 4700 \\ KAUST, Thuwal, Saudi Arabia 21534
}

\begin{abstract}
We reported on the design, demonstration, and analysis of white lighting systems based on GaN laser diodes. Compared to light-emitting-diodes (LEDs), lasers have been proposed for the development of high-power light sources for many potential advantages, including circumventing efficiency droop, reduced light emitting surface, directional beam characteristics. Laser-based white light sources are also attractive for visible light communication (VLC) applications that enabling lighting and communication dual functionalities. In this work, we detailed the color-rendering index (CRI), correlated color temperature (CCT), and luminous flux analysis of laser white light sources by using the GaN laser diode exciting color converters at various driving conditions. By using a blue-emitting laser exciting a yellow YAG phosphor crystal, a luminous flux greater than $600 \mathrm{~lm}$ has been achieved with a moderate CRI of 67.2. By constructing a white lighting system using phosphor crystal array based on a reflection configuration, an improved CRI of 74.4 and a luminous flux of $\sim 400 \mathrm{~lm}$ with a CCT of $6425 \mathrm{~K}$ was obtained at 3A. Using a novel ceramic phosphor plate as color converter, the CRI for the white light source has been further improved to $\sim 84.1$ with a CCT of $\sim 4981 \mathrm{~K}$, which suggests that the laserbased white light source is capable of high-quality illumination applications. The CCT of the white laser sources can be engineered from $5000 \mathrm{~K}$ to $6500 \mathrm{~K}$ and a potential approach to use laser array for high power white lighting is discussed.
\end{abstract}

Keywords: Gallium Nitride, laser diode, semiconductor laser, solid state lighting, light emitting diodes.

\section{INTRODUCTION}

Solid-state lighting (SSL) technology uses semiconductor light emitters, such as light emitting diodes (LEDs), offering a versatile and energy-efficient light source with high color stability and growing lumen efficacy [1-3]. White LED bulbs based on GaN LEDs have been successfully commercialized with the potential to be the dominant standard for general lighting since the cost of LED chips drops in the past decade [4-6]. Eventually, LEDs will likely take over many if not all indoor and outdoor lighting. Besides, LEDs are used for backlighting, display, and visible light communications. Recently, GaN-based laser diode (LD) has attracted increasing attention for enabling novel white light sources [7]. One of the critical driving forces is the potential to build compact, high intensity light bulb using lasers as LDs are not associated with "efficiency droop" effect. In LEDs, a significant drop in external quantum efficiency (EQE) is observed as operating current increases, which is known as "efficiency droop" [8,9]. Since the carrier clamping in the cavity after threshold point of lasing [10], the EQE of LDs linearly increases with increasing operating current [11]. In addition, laser based white light source has many other potential advantages such as minimized light emitting surface, directional beam characteristics, and ultra-fast operation speed, making it promising for future smart lighting applications.

In 2013 and 2014, Wierer et al. and Tsao presented a detailed discussion and review on the advantage and potential of laser-based SSL. Since then, the utilization of laser for white light generation for various applications has been reported. For example, laser based solid-state light source for etendue-limited applications [12], automotive lighting applications [13], indoor lighting and communication (Li-Fi) [14] have been discussed. In particular, since GaN-based LDs have much higher modulation bandwidth than LEDs, LDs are becoming attractive to serve as the light emitter and signal transmitter in visible light communication systems [15-20]. More recently, LD based white light sources have been used for underwater lighting and wireless data communication [21]. Therefore, the study of high-intensity laser-based white lighting systems becomes an important topic for many emerging applications.

*chao.shen@kaust.edu.sa; phone 96612 808-3814; www.sanoortech.com 
In general, there are two approaches to generate white light using LDs, by directly mixing the light from three (or more) monochromatic sources, such as red, green and blue (RGB) LDs, or using violet-blue LD integrated with phosphors. Both approaches have advantages and challenges, but the later scenario is more widely studied due to is relatively simple structure and low cost.

\subsection{Combination of RGB lasers}

By combining red, green and blue laser beams, white light can be generated as illustrated in Figure 1. For smart lighting, the integration of RGB lasers offers great flexibility and enhanced lighting quality for display applications. Yang et al. reported a highly uniform white laser lamp based on RGB lasers [15]. By mixing RGB LDs to generate a white light beam with a correlated color temperature (CCT) of $6500 \mathrm{~K}$, Huang et al. demonstrated a white-light communication system with divergent diffuser optimization [22]. The potential to use RGB laser white light source for high-speed WDM communications in free space and underwater channels were also reported $[16,23]$. However, white light based on combining RGB lasers are costly, partly due to the relatively low efficiency in green LDs and high cost in high power red LDs.
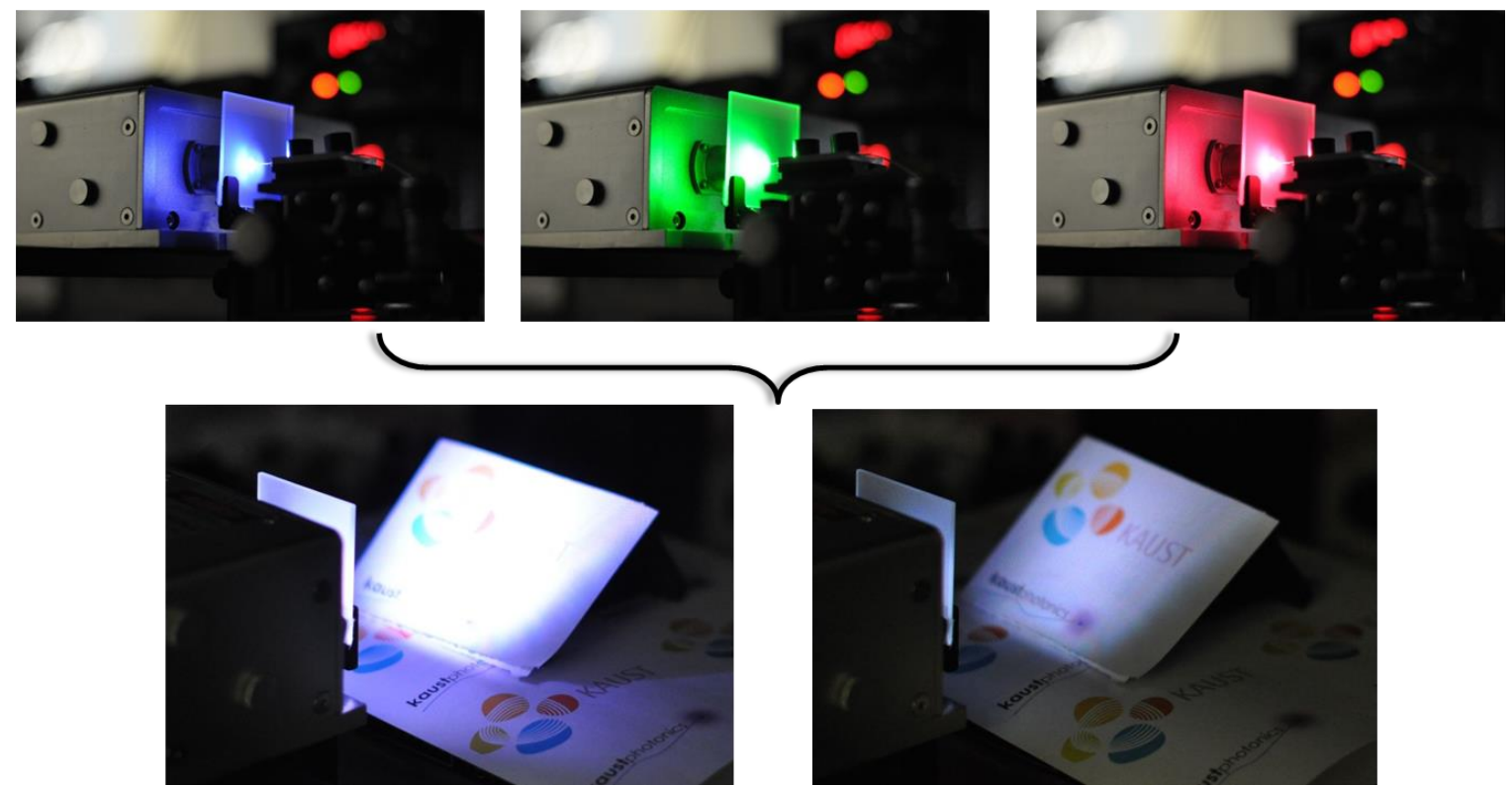

Figure 1. By combining the blue, green and red laser beam, white light source can be built. A diffuser was placed in front of the output to diffuse the white laser beam.

\subsection{Blue laser with phosphors}

Similar to the white LED or SLD lamp, a more practical approach to generate white light is using blue laser to excite yellow phosphors or mixture of two or more phosphors as color converters, as shown in Figure 2 [11, 24]. In 2015, Lee et al. presented a system that uses a blue gallium nitride LD to excite YAG:Ce phosphors to produce white light that has a CRI of 58 and the CCT of the produced light was found to be $4740 \mathrm{~K}$ [25]. Later, a number of research demonstrations have been reported for general illumination, projector, automotive and Li-Fi applications [20, 26-29]. Since the laser based white light bulb has the great potential for high intensity applications, there are growing research in developing color converters for laser based white lighting as the excitation power density is orders of magnitude higher than those in LED white lamps [27, 30-33].

In this work, we designed and demonstrated compact laser-based white light modules and discussed the approaches towards high quality and high power laser light sources. The presented laser white light modules can be configurated as narrow beam emitter for long distance spot lighting and have promising potential to enable high-speed visible light communication links. 


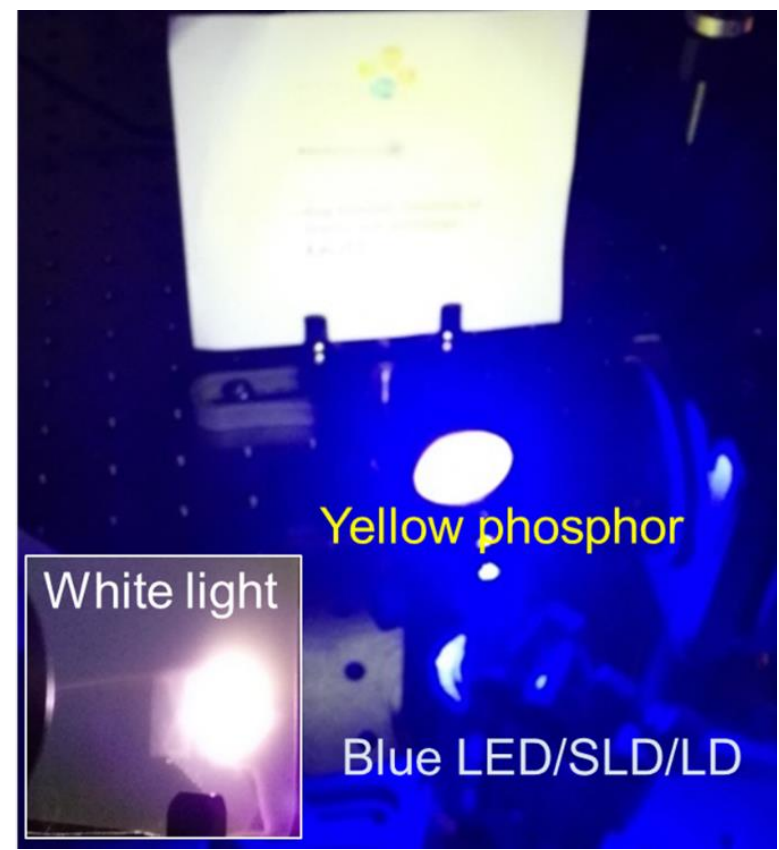

Figure 2. White light generated using blue LD exciting yellow phosphors. Part of the blue light is converted into the yellow portion of the white light after passing through the phosphor material while the unconverted blue light contributes to the blue portion of the white light spectrum.

\section{EXPERIMENTS}

Device 1 (SNWL-1) contains a $450 \mathrm{~nm}$ blue laser diode mounted on brass heatsink with a glass diffuser and a circular YAG:Ce crystal as color converter. The phosphor crystal was installed in front of the laser beam output and the generated white light, which is the combination of the transmitted blue light and the converted yellow emission, was directly emitted out of the device. Device 2 (SNWL-2) is constructed with the same blue laser and an array of square phosphor plates mounted on a brass heatsink with a $45^{\circ}$ inclined plane as reflector. The $2 * 2$ phosphor plate array was mounted on the surface of the slope reflector so white light can be generated in a reflecting configuration. Device 3 (SNWL-3) features a blue laser exciting the ceramic phosphor plate mounted on the brass heat sink using a reflecting configuration. The ceramic phosphor plate contains two phosphor materials, which emitting at green and red bands. Device 4 (SNWL-1a) contains 3 blue lasers mounted in one module with the circular YAG:Ce phosphor crystal. All the devices were tested without active cooling. A current source (Keithley 2420) is used to driver the laser. The testing was done using a $0.5 \mathrm{~m}$ diameter integrating sphere connected to a lamp testing system (HOPOO HP8000). To evaluate the system's performance as an SSL lighting source, the factors considered are Color-Rendering Index (CRI) and Correlated Color Temperature (CCT). CRI evaluates the ability of the produced light to reveal colors while CCT describes the appearance of the white light. The luminous flux, power consumption and optical spectrum are measured at the same time. All the measurements were done at room temperature.

\section{RESULTS AND DISCUSSIONS}

The emission spectrum and CIE chromaticity diagram for device 1 at $3 \mathrm{~A}$ is shown in Figure 3 . The generated cool white light has a CRI of 67.2, CCT of $6391 \mathrm{~K}$ and a total luminous flux of $625 \mathrm{~lm}$. In order to study the consistency of CRI and CCT at different injection currents, the changes of CRI and CCT at increasing injection currents are plotted in Figure 4. Since the laser has a threshold current of approximately $450 \mathrm{~mA}$, the device was measured with injection ranging from $500 \mathrm{~mA}$ to $3 \mathrm{~A}$. The variation of CRI and CCT, i.e. the difference between maximum value and minimum value, is $\sim 7.3$ and $\sim 550 \mathrm{~K}$, respectively. The luminous flux at different injection currents can be found in Figure 5. 


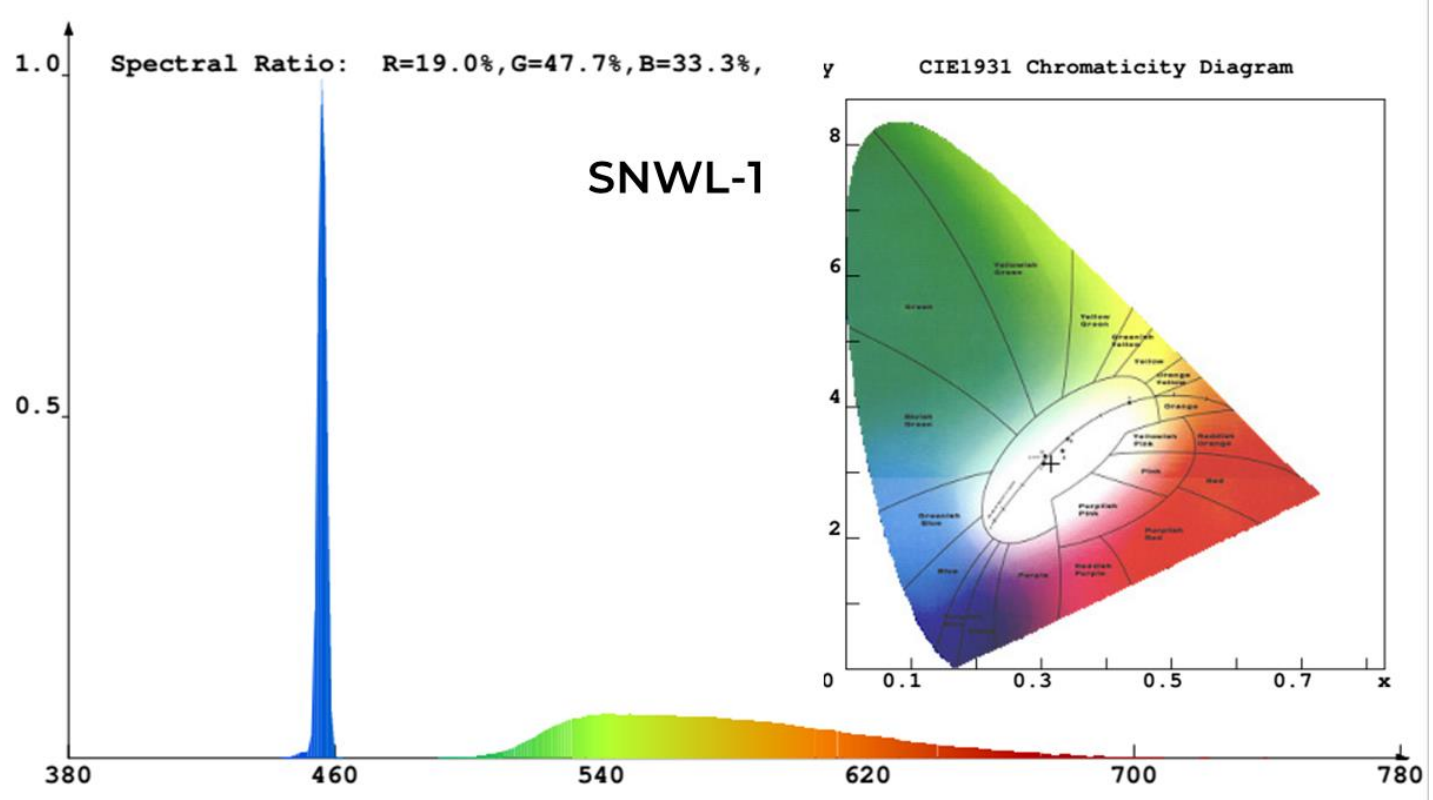

Figure 3. Emission spectrum and the CIE chromaticity diagram for device 1 at a driving current of $3 \mathrm{~A}$.

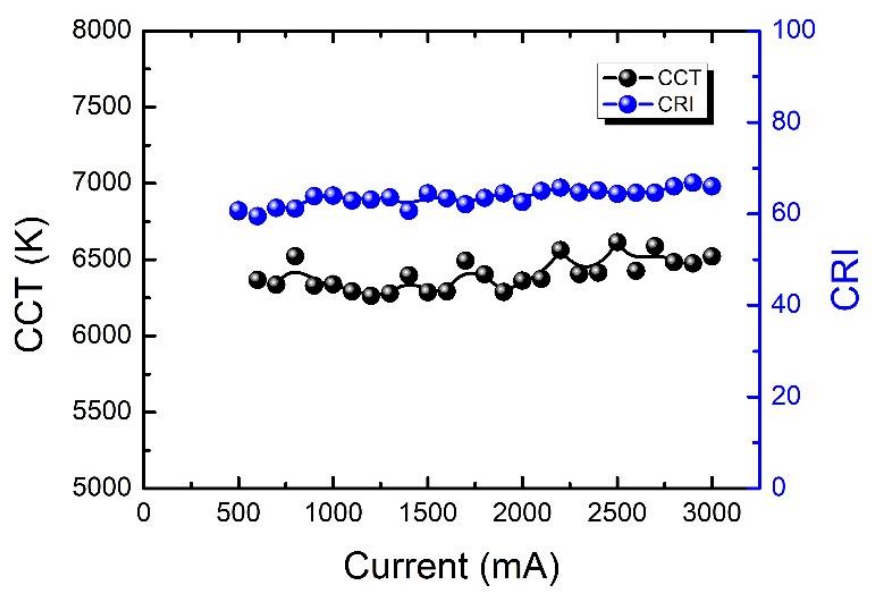

Figure 4. Changes of CCT and CRI as a function of driving current in device 1 (SNWL-1).

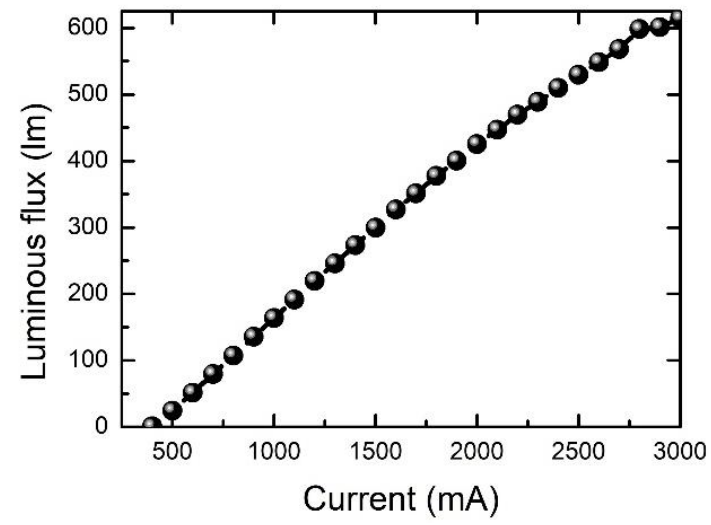

Figure 5. Luminous flux as a function of driving current in device 1 (SNWL-1). 
We performed similar measurements for devices 2 and 3. The emission spectrum and CIE chromaticity diagram for device 2 at $3 \mathrm{~A}$ is shown in Figure 6. Device 2 has a CRI of 74.4, CCT of $6425 \mathrm{~K}$ and a total luminous flux of $401 \mathrm{~lm}$ at $3 \mathrm{~A}$. In this configuration, the CRI has been increased at the cost of lower total luminous flux achieved compared with device 1 . The variation of CRI and CCT is $\sim 5.8$ and $\sim 530 \mathrm{~K}$, respectively, which is slightly improved compared with device 1 . For device 3, the CRI has been increased to 84.1 with a CCT of $4981 \mathrm{~K}$ at $2.5 \mathrm{~A}$. The device shows a very stable CRI performance with a CRI variation as little as 2.9, making it very promising for high quality neutral white lighting applications. The luminous flux of $283 \mathrm{~lm}$ is measured at $2.5 \mathrm{~A}$, which is lower than that of $357 \mathrm{~lm}$ in device 2.

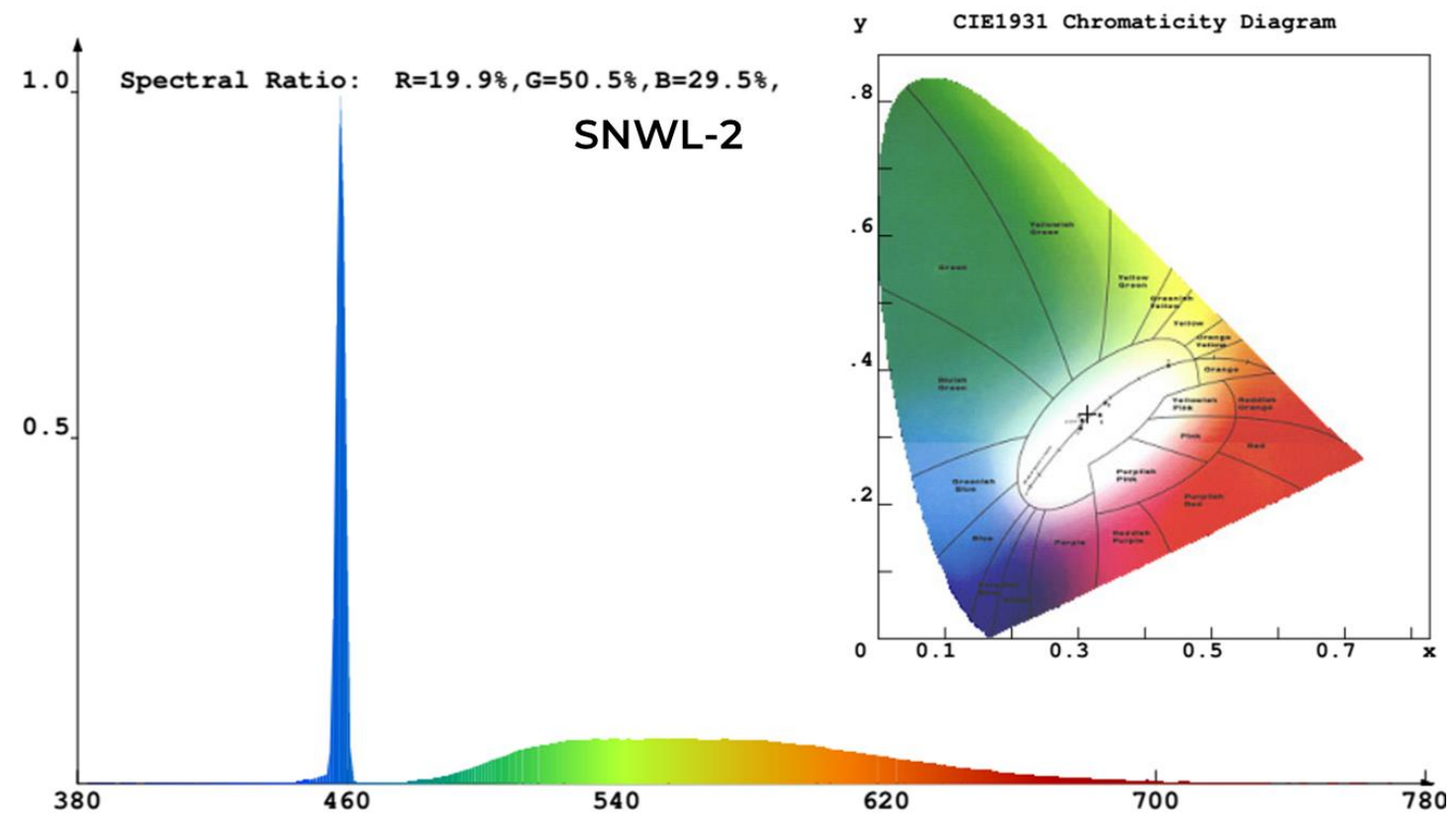

Figure 6. Emission spectrum and the CIE chromaticity diagram for device 2 at a driving current of $3 \mathrm{~A}$.

To achieve high intense laser lighting system, we integrated 3 laser diodes in one device (SNWL-1a). At injection current of 3A, the device emits a total flux of $1606.8 \mathrm{~lm}$. The measured CRI and CCT are 63.9 and $4179 \mathrm{~K}$, respectively. The luminous efficacy at $3 \mathrm{~A}$ is calculated to be $32.6 \mathrm{~lm} / \mathrm{W}$. This result indicates high intensity white light sources can be achieved using integrated GaN laser array configuration. The high-power white laser light sources presented in this work can also serve as the transmitter for high-speed data communications. Utilizing the devices studied, we have achieved a wireless data communication over $1 \mathrm{Gbps}$ using on-off keying modulation technique, suggesting its attractive property for outdoor Li-Fi and long distance underwater optical communication applications.

\section{CONCLUSIONS}

In this paper, the recent progress in laser-based white lighting devices and systems have been discussed. Different designs towards high CRI, high intensity laser white lighting devices are demonstrated and measured. The CRI of laser-based white lighting system can be engineered from 67.2 to 84.1 and the CCT can be tuned from $5000 \mathrm{~K}$ to $6500 \mathrm{~K}$. The luminous flux for a single laser based white lighting device can be as high as $600 \mathrm{~lm}$ and by integrating multiple laser diodes to assemble the laser array, a high-power white lighting system with a total flux exceeding $1600 \mathrm{~lm}$ can be achieved. Such white laser device as a high intensity light source will be useful for many emerging applications, such as outdoor lighting, projection, and high-speed visible light communications.

\section{ACKNOWLEDGEMENT}

The authors acknowledge the financial support from King Abdulaziz City for Science and Technology (KACST) Grant No. KACST TIC R2-FP-008. This publication is based on work supported by the King Abdullah University of Science and Technology (KAUST) (baseline funding, BAS/1/1614-01-01, KAUST funding SEE/1/1808-01-01). 


\section{REFERENCES}

[1] R. Haitz, and J. Y. Tsao, "Solid-state lighting: 'The case' 10 years after and future prospects," Physica Status Solidi a, 208(1), 17-29 (2011).

[2] S. Nakamura, "Current Status of GaN-Based Solid-State Lighting," MRS Bulletin, 34(2), 101-107 (2009).

[3] M. S. Shur, and A. Zukauskas, "Solid-state lighting: Toward superior illumination," Proc. IEEE, 93(10), 16911703 (2005).

[4] S. P. DenBaars, D. Feezell, K. Kelchner et al., "Development of gallium-nitride-based light-emitting diodes (LEDs) and laser diodes for energy-efficient lighting and displays," Acta Materialia, 61(3), 945-951 (2013).

[5] H. Masui, S. Nakamura, S. P. DenBaars et al., "Nonpolar and Semipolar III-Nitride Light-Emitting Diodes: Achievements and Challenges," IEEE Transactions on Electron Devices, 57(1), 88-100 (2010).

[6] G. T. Wang, Q. M. Li, J. Y. Huang et al., "III-nitride nanowires: Novel materials for solid-state lighting," Proc. SPIE, 7954, (2011).

[7] J. J. Wierer, J. Y. Tsao, and D. S. Sizov, “The potential of III-nitride laser diodes for solid-state lighting,” Physica Status Solidi C, 11(3-4), 674-677 (2014).

[8] G. Verzellesi, D. Saguatti, M. Meneghini et al., "Efficiency droop in InGaN/GaN blue light-emitting diodes: Physical mechanisms and remedies," Journal of Applied Physics, 114(7), 071101 (2013).

[9] D. S. Meyaard, G. B. Lin, J. Cho et al., "Identifying the cause of the efficiency droop in GaInN light-emitting diodes by correlating the onset of high injection with the onset of the efficiency droop," Applied Physics Letters, 102(25), 251114 (2013).

[10] J. J. Wierer, J. Y. Tsao, and D. S. Sizov, "Comparison between blue lasers and light-emitting diodes for future solid-state lighting," Laser \& Photonics Reviews, 7(6), 963-993 (2013).

[11] K. A. Denault, M. Cantore, S. Nakamura et al., "Efficient and stable laser-driven white lighting," AIP Advances, 3(7), 072107 (2013).

[12] N. Abu-Ageel, and D. Aslam, "Laser-Driven Visible Solid-State Light Source for Etendue-Limited Applications," Journal of Display Technology, 10(8), 700-703 (2014).

[13] C. Altingoz, "Laser technology in automotive lighting," Proc. SPIE, 8965 (2014).

[14] Y. C. Chi, D. H. Hsieh, C. Y. Lin et al., "Phosphorous Diffuser Diverged Blue Laser Diode for Indoor Lighting and Communication," Scientific Reports, 5, 18690 (2015).

[15] J. Yang, Z. Liu, B. Xe et al., "Highly Uniform White Light-Based Visible Light Communication Using Red, Green, and Blue Laser Diodes," IEEE Photonics Journal, 10(2), 8200508 (2018).

[16] T.-C. Wu, Y.-C. Chi, H.-Y. Wang et al., "Tricolor R/G/B Laser Diode Based Eye-Safe White Lighting Communication Beyond 8 Gbit/s," Scientific Reports, 7(1), 11 (2018).

[17] S. Watson, S. Viola, G. Giuliano et al., "High speed visible light communication using blue GaN laser diodes." Proc. SPIE, 99910A-99910A-7 (2016).

[18] C. Lee, C. Shen, H. M. Oubei et al., "2 Gbit/s data transmission from an unfiltered laser-based phosphor-converted white lighting communication system," Opt Express, 23(23), 29779-87 (2015).

[19] C. Lee, C. Shen, C. Cozzan et al., "Semipolar GaN-based laser diodes for Gbit/s white lighting communication: devices to systems." Proc. SPIE 10532, 11 (2017).

[20] C. Lee, C. Shen, C. Cozzan et al., "Gigabit-per-second white light-based visible light communication using nearultraviolet laser diode and red-, green-, and blue-emitting phosphors," Optics Express, 25(15), 17480-17487 (2017).

[21] X. Y. Liu, S. Y. Yi, X. L. Zhou et al., "Laser-based white-light source for high-speed underwater wireless optical communication and high-efficiency underwater solid-state lighting," Optics Express, 26(15), 19259-19274 (2018).

[22] Y. F. Huang, Y. C. Chi, M. K. Chen et al., "Red/green/blue LD mixed white-light communication at 6500K with divergent diffuser optimization," Optics Express, 26(18), 23397-23410 (2018).

[23] M. W. Kong, W. C. Lv, T. Ali et al., "10-m 9.51-Gb/s RGB laser diodes-based WDM underwater wireless optical communication," Optics Express, 25(17), 20829-20834 (2017).

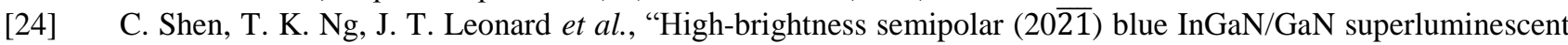
diodes for droop-free solid-state lighting and visible-light communications," Opt Lett, 41(11), 2608-11 (2016).

[25] C. Lee, C. Shen, H. M. Oubei et al., "2 Gbit/s data transmission from an unfiltered laser-based phosphor-converted white lighting communication system,” Optics Express, 23(23), 29779-29787 (2015). 
[26] S. Y. Tu, H. Y. Lin, and T. X. Lee, "Efficient speckle-suppressed white light source by micro-vibrated and colormixing techniques for lighting applications," Optics Express, 23(20), 26754-26768 (2015).

[27] A. F. George, S. Al-Waisawy, J. T. Wright et al., "Laser-driven phosphor-converted white light source for solidstate illumination," Applied Optics, 55(8), 1899-1905 (2016).

[28] Y. H. Song, E. K. Ji, B. W. Jeong et al., "High power laser-driven ceramic phosphor plate for outstanding efficient white light conversion in application of automotive lighting," Sci Rep, 6, 31206 (2016).

[29] F. Aquino, W. M. Jadwisienczak, and F. Rahman, "Effect of laser speckle on light from laser diode-pumped phosphor-converted light sources," Applied Optics, 56(2), 278-283 (2017).

[30] S. X. Li, L. Wang, N. Hirosaki et al., "Color Conversion Materials for High-Brightness Laser-Driven Solid-State Lighting," Laser \& Photonics Reviews, 12(12), (2018).

[31] S. X. Li, D. M. Tang, Z. F. Tian et al., "New insights into the microstructure of translucent CaAlSiN3:Eu2+ phosphor ceramics for solid-state laser lighting," Journal of Materials Chemistry C, 5(5), 1042-1051 (2017).

[32] C. Cozzan, M. J. Brady, N. O'Dea et al., "Monolithic translucent $\mathrm{BaMgAl}_{10} \mathrm{O}_{17}: \mathrm{Eu}^{2+}$ phosphors for laser-driven solid state lighting," Aip Advances, 6(10), (2016).

[33] S. Al-Waisawy, W. M. Jadwisienczak, J. T. Wright et al., "Laser excitation of red, green, blue and trichromatic white rare-earth phosphors for solid-state lighting applications," Journal of Luminescence, 169, 196-203 (2016). 